\title{
Interdisciplinary Treatment for Cutaneous Abdominal Wall Metastasis from Cervical Cancer with Resection and Reconstruction of the Abdominal Wall Using Free Latissimus Dorsi Muscle Flap: A Case Report
}

Fallbericht einer komplexen interdisziplinären Behandlung kutaner Bauchwandmetastasen beim Zervixkarzinom durch Resektion und Bauchdeckenrekonstruktion mit freier Latissimus-dorsi-Muskellappenplastik

Authors

Affiliations
A. M. Boos ${ }^{1}$, M. W. Beckmann ${ }^{2}$, R. E. Horch ${ }^{1}$, J. P. Beier ${ }^{1}$

${ }^{1}$ Department of Plastic and Hand Surgery, University Hospital of Erlangen, Erlangen

2 Department of Obstetrics and Gynecology, University Hospital of Erlangen, Erlangen
Deutschsprachige Zusatzinformationen online abrufbar unter: www.thieme-connect.de/ ejournals/toc/gebfra

received 26.1.2014 revised $\quad 9.3 .2014$ accepted 11.3.2014

Bibliography

DOI http://dx.doi.org/

10.1055/s-0034-1368425

Geburtsh Frauenheilk 2014; 74 :

574-578 @ Georg Thieme

Verlag KG Stuttgart · New York . ISSN 0016-5751

\section{Correspondence}

Dr. Justus P. Beier, M. D.

Universitätsklinikum Erlangen, Friedrich-Alexander-Universität Erlangen-Nürnberg

Plastisch- und Handchirurgische Klinik

Krankenhausstraße 12

91054 Erlangen

justus.beier@uk-erlangen.de

\section{Introduction}

$\nabla$

Despite extensive screening and a significant increase in the numbers of diagnosed and treated precancerous lesions, cervical cancer is the second most common cancer in women. Screening programs have resulted in a drop in the number of women newly diagnosed with cervical cancer every year, and many tumors are detected in their early stages [1]. Vaccinations against different human papilloma viruses only became generally available a few years ago, and most women between the age of 30 and 40 years have therefore not benefitted from the protection afforded by vaccination [2]. Early lymphatic metastasis often occurs in the parametrium and the pelvic lymph nodes. Hematogenous dissemination tends to occur relatively late and commonly involves the liver, lungs and skeleton [3]. Cutaneous metastases are found in fewer than $2 \%$ of patients and are usually associated with a poor prognosis [4]. Straightforward plastic surgery with reconstruction using advancement or rotation flaps obtained from the area around the defect are unsuitable in these cases because the recipient site has been treated with radiotherapy, which is likely to increase the problem. Microsurgical free-tissue transfer uses healthy, non-irradiated, well-vascularized tissue. There are very few reports of abdominal wall recurrence of the type described here, and their treatment, particularly if local flap plasty results in further skin and soft-tissue defects, represents a challenge for an interdisciplinary team of gynecologists and general and plastic surgeons. In addition to covering the defect, the aim of surgery is either to preserve the prosthetic mesh required for stabilization or to create an alternative means of stabilization. The treatment algorithm used in this interdisciplinary approach is described for a patient with this rare recurrence.

\section{Case Report - Early History \\ $\nabla$}

During regular screening, a 34-year-old patient with two previous uncomplicated pregnancies and births was diagnosed with squamous cell cancer of the cervix (pT2b, pN1 [2/29] G3 pL1 pV1 pR1, anterior cervical wall/left parametrium, stage IIb). The patient underwent a WertheimMeigs extended radical hysterectomy with preservation of the adnexa and pelvic lymphadenectomy. The patient subsequently underwent radiation therapy of the pelvis which included the lymphatic drainage area (dose: $50.4 \mathrm{~Gy}$ ) with a booster dose delivered to the parametrium (total dose: $59.4 \mathrm{~Gy}$ ) and an afterload dose of $5 \mathrm{~Gy}$ administered intravaginally. The patient received simultaneous chemotherapy with cisplatin, $1 \times$ week for 6 weeks.

One and a half years after the primary diagnosis, abdominal wall recurrence in the vicinity of the laparotomy scar was diagnosed as an incidental finding during scar and wound revision. This was treated by wide resection. Histopathological examination showed a moderately differentiated, focally keratinizing squamous cell carcinoma with tumor which had spread to the soft tissue resection margin (pR1 G2-3). Oncological treatment at the time was done at another hospital and consisted of non-R0 resection, followed by combined radiation and chemotherapy with radiation therapy of the periumbilical abdominal wall up to the mons pubis (dose: $50.4 \mathrm{~Gy}$ ), a booster up to a dose of $55.8 \mathrm{~Gy}$ and cisplatin chemotherapy for 6 weeks. After a recurrence-free interval, metastasis of the abdominal wall was detected again 3 years after the primary diagnosis, whereupon the patient again underwent non-complete resection at another hospital, resulting 6 months later in yet another abdominal wall recurrence, upon which the patient presented to our hospital. 


\section{Abdominal Wall Recurrence after Squamous Cell Cancer of the Cervix} $\nabla$

The case was presented to our interdisciplinary tumor board. Multiple punch biopsies were done to define the tumor-free margins of the recurrence ( Fig. 1a). Laparoscopy was performed to exclude intraabdominal/intraperitoneal tumor seeding. After interdisciplinary discussion of the case, the recurrence was completely resected without complications. Resection consisted of bilateral resection of the anterior lamina of the rectus sheath. A double Prolene mesh was implanted to reinforce the abdominal wall and primary wound closure was done using a local expanded flap with mobilization of the abdominal wall like an abdominoplasty with umbilical resection. Postoperatively, the patient presented with wound edge necrosis and increasing demarcation. Subsequent to debridement, a VAC sponge was placed temporarily to treat the wound ( Fig. $\mathbf{1 b}$ ). Debridement and cleaning of the wound resulted in exposure of the Prolene mesh with a soft tissue defect in the lower abdomen, extending cranially from the former demarcation line to the pubic symphysis caudally and measuring approx. $20 \times 30 \mathrm{~cm}$. The mesh was exposed almost across the entire surface area ( $\bullet$ Fig. $1 \mathrm{c}$ ).

\section{Defect Coverage with Free Muscle Flap Plasty \\ $\nabla$}

When the patient first presented to our Department of Plastic and Hand Surgery, examination showed a defect of the abdominal wall with exposure of the mesh which had been used to reinforce the abdominal wall after resection of the anterior lamina of the rectal sheath. An interdisciplinary team of gynecologists and general and plastic surgeons studied the findings and proposed abdominal wall revision with mesh explantation and reconstruction of the defect with free latissimus dorsi muscle flap.

Preoperative computed tomography/angiography of the abdominopelvic region showed no evidence of local recurrence.

Defect reconstruction began with explantation of the exposed Prolene mesh by the general surgeon and included wound margin biopsies for quick section diagnosis ( $\bullet$ Fig. 2a). Rapid histological examination found no evidence of malignancy. Based on the evaluation of the general surgeon, the abdominal wall appeared sufficiently stable without requiring implantation of a new mesh. Defect coverage and closure was done by free microsurgical myocutaneous latissimus dorsi flap plasty with end-toside arterial anastomosis to the femoral artery and a venous coupler for anastomosis to the left great saphenous vein. The muscle flap was covered by a split skin graft obtained from the thigh and a VAC sponge was applied. Perfusion of the flap was initially checked every hour, then every second hour using a monitoring skin island ( Fig. 2 b). Perfusion was normal at all times.

The monitoring island was experienced as troublesome by the patient when flexing her hip joint and was removed after one week. The ensuing defect was covered by split-thickness skin graft from the monitoring skin island. The postoperative course was unremarkable except for a small wound healing disorder on the left side lateral to the flap plasty ( $\bullet$ Fig. $\mathbf{3}$ b). It was treated conservatively and healed without complications. Another small wound healing disorder developed on the patient's back which also healed quickly after conservative wound treatment ( $\bullet$ Figs. 3 a and c).
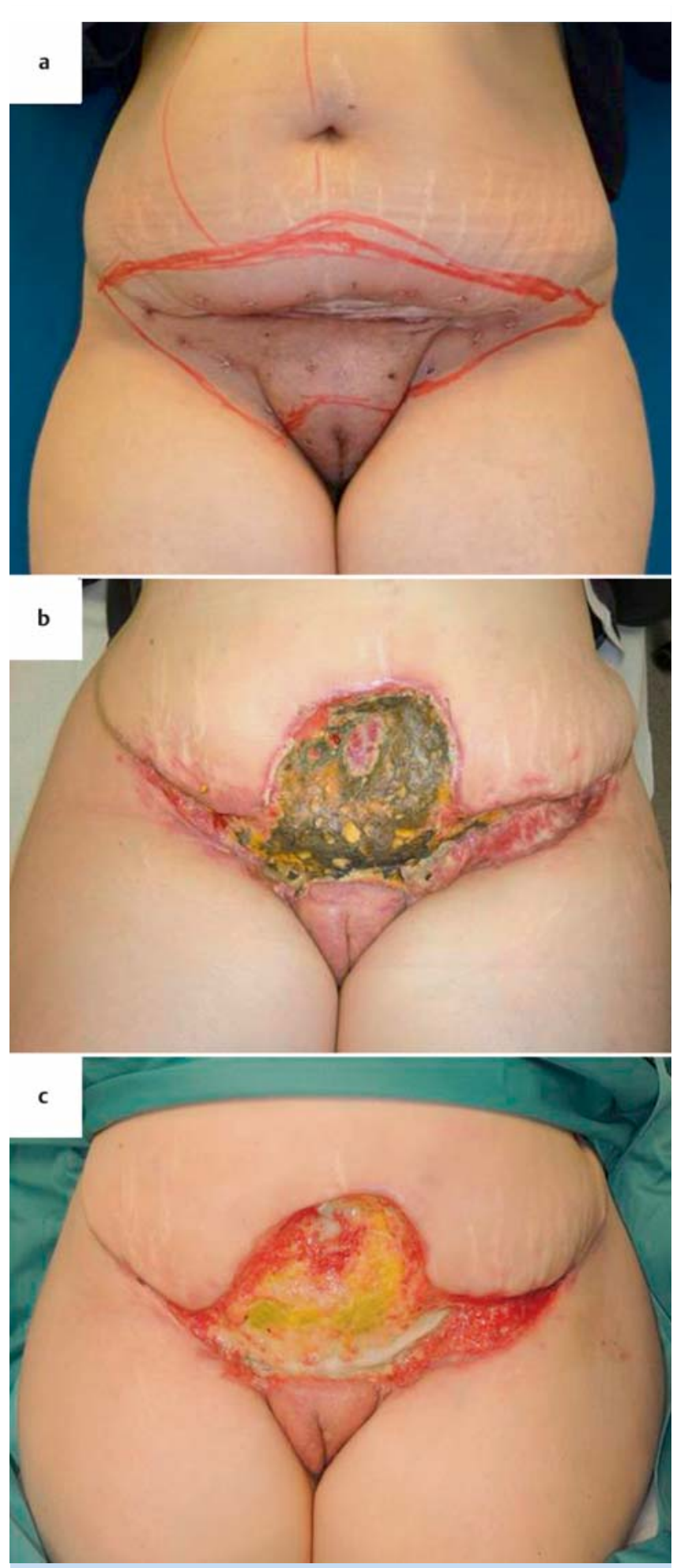

Fig. 1 a to $\mathbf{c}$ a Third recurrence in the abdominal wall after squamous cell carcinoma of the cervix. After punch biopsy and determination of the resection margins. b After wound healing disorder subsequent to repeated VAC treatment and wound demarcation. c After treatment of the open wound and the exposed mesh with daily rinsing, prior to undergoing more debridement and coverage of the defect. 


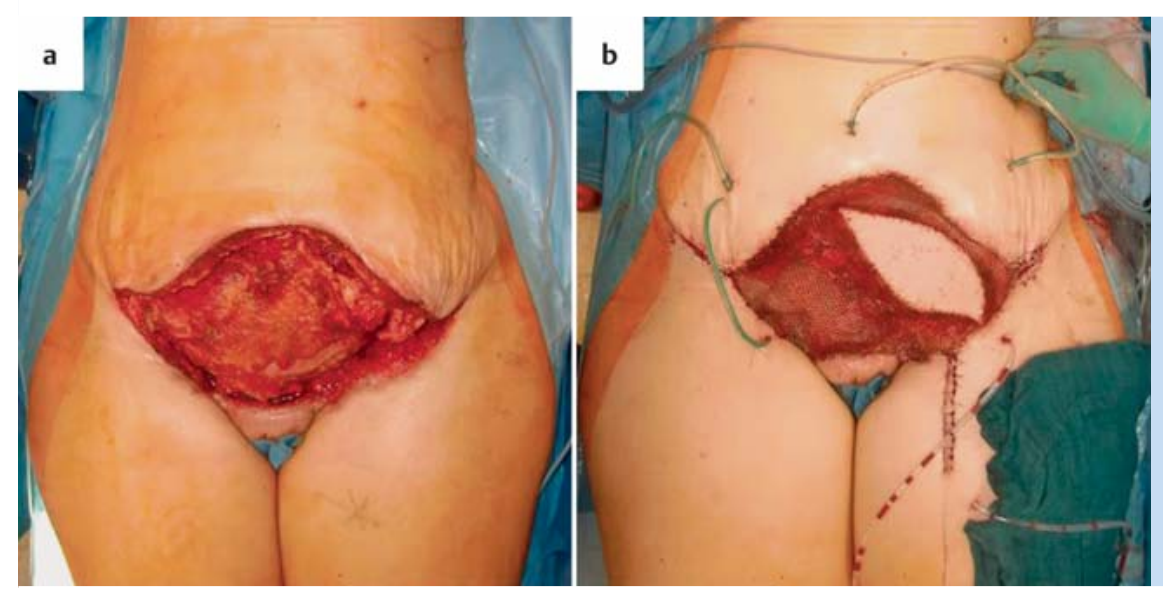

Fig. $2 \mathrm{a}$ and $\mathbf{b}$

a Defect after radical wound debridement with resection of the anterior lamina of the rectal sheath and removal of the exposed mesh used to reinforce the abdominal wall.

b Defect coverage with free myocutaneous latissimus dorsi flap and microsurgical end-to-side arterial anastomosis to the femoral artery and a coupler for venous anastomosis to the left great saphenous vein. The muscle flap was covered by meshed splitthickness skin graft from the thigh. A skin island to monitor perfusion was temporarily sewn on.

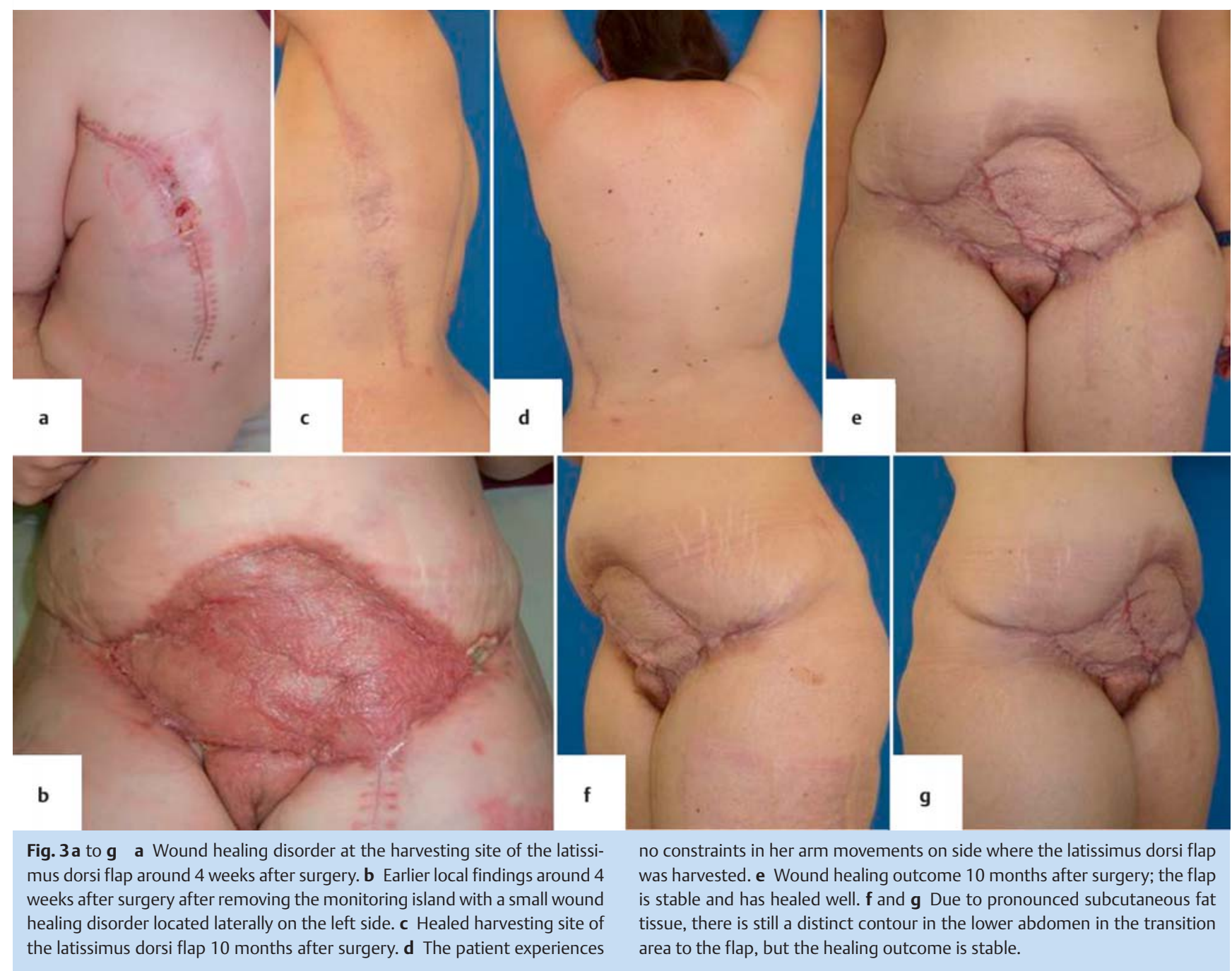

\section{Follow-up}

$\nabla$

The patient comes for regular follow-up examinations to the Gynecological Department and the Plastic and Hand Surgery Department. At 12 months after surgery, the patient remains free of recurrence and there has been no evidence of further metastasis or repeat recurrence in the abdominal wall. The flap and the transplanted skin-thickness graft are well perfused, and after healing the areas are fully functional ( $\odot$ Fig. $\mathbf{3}$ e to g). Due to pronounced subcutaneous fat tissue, there is still a distinct contour in the lower abdomen in the transition area to the flap but the healing outcome is stable. At the start, great care was taken not to place too much stress on the abdominal wall. The patient avoided lifting any heavy loads weighing more than $5 \mathrm{~kg}$ for a period of 10 weeks after surgery and wore an abdominal belt. No 
abdominal wall weakness or hernia was noted during the followup period of 1 year.

The scar on the patient's back healed well and has already faded. The patient has been careful to carry out intensive scar care with lipid-replenishing creams and silicone-containing scar ointments. The patient experiences no constraints with regard to arm movements on the side where the flap was harvested ( Fig. 3d). The patient has only reported a slight reduction in movement when twisting her torso and during maximum flexion, which is perceptible as pulling and tension on the muscles, but this has already decreased with physiotherapy and scar massage. After this extensive soft tissue reconstruction the patient is able to carry out all daily activities with almost no restrictions and is able to care for her children.

\section{Discussion}

Metastasis of the abdominal wall after cervical cancer is a rare occurrence in the follow-up of tumor patients and represents a challenge for the interdisciplinary team of gynecologists and general and plastic surgeons [5]. The majority of treatments described in the literature consist predominantly of radiation and chemotherapy with local excision of metastases [6]. One case report describes a patient with cutaneous lymphangitis carcinomatosa who received palliative chemotherapy [7].

Skin and soft tissue resection is often possible without any difficulty as there is sufficient skin and soft tissue in the lower abdomen. However, if the patient has undergone prior several operations or has previously had adjuvant therapy, as was the case with our patient who had undergone radiation therapy (twice) previously, this may negatively affect local conditions. Moreover, it should be noted critically for this patient that, during initial treatment at an external hospital, no R0 resection was achieved. This can negatively affect the prognosis for overall and local recurrence and impacts directly on the necessity for adjuvant treatment. Patients who have had several cycles of radiation therapy may go on to develop substantial wound healing deficits and necrosis which may preclude sufficient local tissue being available for coverage and require tissue transplantation from other sites. In the case described here, tissue from the surrounding soft tissue area had already been mobilized. The external aspect clearly shows the permanent damage done by repeated radiation therapy ( Fig. 1). Moreover, a mesh had been implanted to reinforce the abdominal wall, and this mesh required explantation subsequently due to infection. It was therefore no longer possible to use simple split-thickness graft or local pedicled flap plasty for defect coverage. Consequently it was necessary to use easily transferrable, free vascularized muscle tissue. The free flap plasty allowed tissue which had not suffered previous injury to be transferred to the irradiated area.

Free muscle flap plasties have been used since many decades to treat similar skin and soft tissue defects at different sites in the body. All reconstructive surgery of the abdominal wall must aim to restore functional stability and provide sufficient soft tissue coverage. Different approaches have been described in the literature. The pedicled tensor fasciae latae muscle flap has been used to recreate fascia continuity and prevent hernia recurrence in patients with extensive fascia defects who have suffered repeated hernia recurrence without an anatomically accessible hernia orifice. An innervated, microsurgically revascularized latissimus dorsi muscle flap can be used to achieve optimal functionality and for esthetic repair of extensive abdominal wall defects affecting all anatomical layers [8]. After healing and reinnervation, a microsurgically anastomosed latissimus dorsi flap can even assume some of the functions of the abdominal wall muscles in patients who have suffered a total loss of abdominal wall muscles [9]. This was not necessary in our patient in whom abdominal wall muscles could be preserved. Use of a perforator-based monitoring skin island in latissimus dorsi flap plasty was first described in 2012 [10]. It was suggested that this could do away with the necessity for a second split-thickness skin graft when removing the monitoring skin island. Unfortunately, in our patient, no suitable perforator was available and the monitoring skin island had be raised using the classical technique and removed later in a second procedure which was followed by repeat split-thickness skin graft. Free latissimus dorsi muscle flaps are also used in the abdomen together with biological meshes to treat complicated hernias. Because of its complexity, this approach is not used routinely in hernia repair but can be a salvage option when treating selected at-risk patients [11]. The latissimus dorsi flap was already used in 1993 together with a Marlex ${ }^{\circledR}$ mesh to close a defect in a patient with cervical cancer and extensive abdominal wall metastasis. The inferior epigastric artery and the external iliac vein were used as the principle anastomoses [12]. As the quality of the vasculature in regions previously treated with radiation therapy is often poor due to radiation damage, the principle anastomoses were chosen from areas outside the irradiated region. Another case report describes a patient with an infected abdominal wall defect from a gunshot wound who underwent reconstruction of the abdominal wall using a polypropylene mesh and free latissimus dorsi muscle flap coverage with an interposition graft and anastomosis to the femoral artery and the great saphenous vein [13]. Because of the good vascularization this approach can also be used to treat large infected abdominal wall defects. In patients with cervical cancer, muscle flap plasty is also used to reconstruct the pelvic floor or vagina and for fistula closure [14]. Unilateral or bilateral pedicled myocutaneous vertical rectus abdominis muscle flaps have been used to close vesicovaginal fistulas [15]. Myocutaneous gracilis flap plasty has been successfully used to reconstruct vulvovaginal defects and fistulas [16]. The possibility of using free muscle plasty to close skin and soft tissue defects should be considered as a therapeutic option in patients with advanced cervical cancer who have had to undergo extensive surgical resection.

\section{Conclusion}

In some rare cases, advanced cervical cancer or its recurrence can involve the abdominal wall. Previously, such cases were usually treated with radiation therapy and chemotherapy as it was thought that extensive resection was too risky because of the exposed structures this would involve. But as microsurgical techniques have continued to evolve and improve, microsurgical free muscle plasty carried out in high volume centers represents a safe plastic surgery procedure for defect closure and could significantly expand the range of extensive local resections by gynecologists. If extensive resection is planned, an interdisciplinary approach involving gynecologists and general and plastic surgeons is important during the early stages of planning as this can help achieve optimal results for the patient with the best possible quality of life and the best chances for long-term survival. 


\section{Conflict of Interest}

$\nabla$

None.

\section{References}

1 Beckmann MW, Mallmann P. Interdisciplinary S2 k guideline on the diagnosis and treatment of cervical carcinoma. J Cancer Res Clin Oncol 2009; 135: 1197-1206

2 De Vincenzo R, Ricci C, Conte $C$ et al. HPV vaccine cross-protection: highlights on additional clinical benefit. Gynecol Oncol 2013; 130: 642-651

3 Massad LS, Einstein MH, Huh WK et al. 2012 updated consensus guidelines for the management of abnormal cervical cancer screening tests and cancer precursors. Obstet Gynecol 2013; 121: 829-846

4 Agrawal A, Yau A, Magliocco A et al. Cutaneous metastatic disease in cervical cancer: a case report. J Obstet Gynaecol Can 2010; 32: 467

5 Mangler M, Zech N, Schneider A et al. Aspects of therapy for cervical cancer in Germany 2012 - results from a survey of German gynaecological hospitals. Geburtsh Frauenheilk 2013; 73: 227-238

6 Juhasz-Boess I, Mallmann P, Moeller CP et al. Stellenwert der Laparoskopie in der Therapie der Endometrium- und Zervixkarzinome - Ergebnisse der AGE \& AGO Klinikumfrage 2012. Geburtsh Frauenheilk 2013; 73: 911-917

7 Palaia I, Angioli R, Cutillo G et al. Skin relapse from cervical cancer. Gynecol Oncol 2002; 87: 155-156

8 Harpf C, Ninkovic M, Kronberger $P$ et al. [Dynamic and functional abdominal wall stabilization, reconstruction of extensive abdominal wall defects]. Chirurg 1997; 68: 488-492
9 Ninkovic M, Kronberger P, Harpf C et al. Free innervated latissimus dorsi muscle flap for reconstruction of full-thickness abdominal wall defects. Plast Reconstr Surg 1998; 101: 971-978

10 Beier JP, Horch RE, Dragu A et al. Perforator-based monitoring skin islands in free muscle flaps: teaching old dogs new tricks. Plast Reconstr Surg 2012; 129: 586e-587e

11 Rauchfuss F, Bader RD, Dittmar Y et al. [The latissimus dorsi myocutaneous free flap-a salvage option for abdominal wall reconstruction in complex incisional hernias]. Handchir Mikrochir Plast Chir 2012; 44: 93-96

12 Neven P, Shepherd JH, Tham KF et al. Reconstruction of the abdominal wall with a latissimus dorsi musculocutaneous flap: a case of a massive abdominal wall metastasis from a cervical cancer requiring palliative resection. Gynecol Oncol 1993; 49: 403-406

13 Bulic K, Dzepina I, Mijatovic D et al. Prosthetic mesh for infected abdominal wall defects? Report of a patient with a large full thickness abdominal wall defect and colostomy due to a gunshot wound. J Plast Reconstr Aesthet Surg 2008; 61: 455-458

14 Gadducci A, Tana R, Cosio $S$ et al. Treatment options in recurrent cervical cancer (Review). Oncol Lett 2010; 1: 3-11

15 Horch RE, Gitsch G, Schultze-Seemann W. Bilateral pedicled myocutaneous vertical rectus abdominus muscle flaps to close vesicovaginal and pouch-vaginal fistulas with simultaneous vaginal and perineal reconstruction in irradiated pelvic wounds. Urology 2002; 60: 502-507

16 Soper JT, Rodriguez G, Berchuck A et al. Long and short gracilis myocutaneous flaps for vulvovaginal reconstruction after radical pelvic surgery: comparison of flap-specific complications. Gynecol Oncol 1995; 56: $271-275$ 